\title{
Analysis of anisotropically permeable surfaces for turbulent drag reduction
}

\author{
Nabil Abderrahaman-Elena and Ricardo García-Mayoral* \\ Department of Engineering, University of Cambridge, Cambridge CB2 1PZ, United Kingdom
}

(Received 27 February 2017; published 30 November 2017)

\begin{abstract}
The present work proposes the use of anisotropically permeable substrates as a means to reduce turbulent skin friction. We conduct an a priori analysis to assess the potential of these surfaces, based on the effect of small-scale surface manipulations on near-wall turbulence. The analysis, valid for small permeability, predicts a monotonic decrease in friction as the streamwise permeability increases. Empirical results suggest that the drag-reducing mechanism is however bound to fail beyond a certain permeability. We investigate the development of Kelvin-Helmholtz-like rollers at the surface as a potential mechanism for this failure. These rollers, which are a typical feature of turbulent flows over permeable walls, are known to increase drag and their appearance is known to limit the drag-reducing effect. We propose a model, based on linear stability analysis, that predicts the onset of these rollers for sufficiently large permeability and allows us to bound the maximum drag reduction that these surfaces can achieve.
\end{abstract}

DOI: 10.1103/PhysRevFluids.2.114609

\section{INTRODUCTION}

In the present work, we carry out an analysis of coatings with anisotropic permeability devised to reduce turbulent skin friction. We explore the possibility of using such coatings to produce the apparent slip that riblets [1] or superhydrophobic surfaces [2] produce to reduce turbulent drag. Hahn et al. [3] found that, in a similar fashion, streamwise-permeable walls reduce skin friction by creating an effective slip for the overlying turbulent flow. In their study, the walls were permeable in the streamwise and spanwise directions only and impermeable in the wall-normal direction. They observed that high values of drag reduction were obtained with low or no spanwise permeability. Intuitively, the surface would then offer less resistance to longitudinal flow than to transverse flow, as riblets do [4]. Real materials cannot however be permeable in only certain directions, but they can have a preferential direction with higher permeability. The seal fur studied by Itoh et al. [5] is, at least in part, an example of such anisotropically permeable material, as it is made up of fibers aligned preferentially in the streamwise direction. Such substrates produce a higher resistance for the crossflow compared to the streamwise flow and indeed Itoh et al. [5] reported drag reduction properties similar to those of riblets, albeit slightly superior, as shown in Fig. 1. Previous studies have shown how a surface can reduce turbulent drag if it induces a streamwise-preferential effect $[4,6,7]$. Taking some length scale of the surface texture as reference, the drag reduction increases linearly with that length, when scaled in viscous units.

The theoretical framework developed in $[4,6,7]$ assumes that the texture is vanishingly small compared to the length scales in the flow. However, as the texture size increases, deleterious effects are experimentally observed and the performance saturates, as studied for riblets by García-Mayoral and Jiménez [8] or for superhydrophobic surfaces by Seo et al. [9] and García-Mayoral et al. [10]. The mechanisms for the onset of the deleterious effects vary, leading to more or less abrupt failures. These failures also occur for different texture sizes, depending on the type of surface.

In permeable substrates, one possible mechanism for the breakdown of drag reduction is the appearance of spanwise coherent rollers over the surface. These are typical of turbulent flows over permeable surfaces when the permeability is large enough [11-15]. While a substrate with a

\footnotetext{
*r.gmayoral@eng.cam.ac.uk
} 


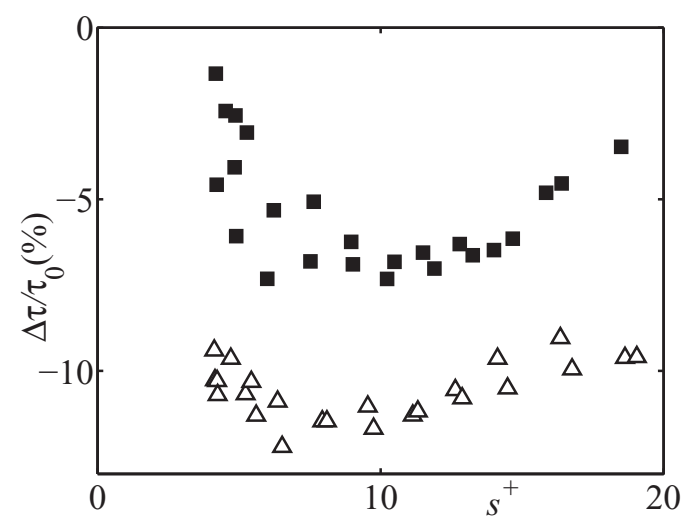

FIG. 1. Drag reduction $D R=-\Delta \tau / \tau_{0}$ of the seal fur surface from Itoh et al. [5]: $\Delta$, seal fur; $\mathbf{\square}$, trapezoidal flat-peak riblets, also from Itoh et al. [5]. For the seal fur, the wavelength identified by Itoh et al. by analogy with the riblet pitch $s^{+}$has been used to express their results in terms of a viscous length scale.

preferential streamwise permeability might yield the drag-reducing effect of Hahn et al. [3] and Itoh et al. [5], permeable surfaces have generally been reported to increase turbulent drag. This was the case in the direct numerical simulation studies of Jiménez et al. [11], where the wall was permeable in the wall-normal direction only, and of Breugem et al. [12], where a substrate of packed particles with isotropic permeability was studied. In both cases, the large increase in drag was associated with the appearance of Kelvin-Helmholtz-like rollers, which enhance momentum transfer and increase the Reynolds stresses near the wall [8]. It is worth noting that Jiménez et al. [11] found that wall-normal permeability alone could trigger the appearance of spanwise rollers and increase drag. This suggests a competition between the beneficial drag-reducing effect, driven by the longitudinal permeability, and the detrimental appearance of Kelvin-Helmholtz-like rollers, driven by the wall-normal permeability. The relaxation of the zero wall-normal velocity condition in complex surfaces is generally responsible for the appearance of nonzero tangential Reynolds stress, increased mixing, and thus increased friction [16,17]. Kelvin-Helmholtz-like rollers appear frequently over complex substrates [18-20] and are indeed a common feature over a wide range of obstructing surfaces [21]. In riblets, they have been identified as the cause for the degradation of performance beyond the linear drag reduction regime, due to the additional Reynolds stresses that they generate [8]. The resemblance of the drag curves for riblets and for the surface of Itoh et al. [5], shown in Fig. 1, could suggest that the degradation is due to a similar mechanism. This work aims to characterize the linearly increasing drag reduction of surfaces with anisotropic permeability, but also to provide an upper bound, based on the development of Kelvin-Helmholtz-like rollers, for what would otherwise be an ever-increasing performance. Our study does not rule out the appearance of additional degrading phenomena, which could be triggered at smaller permeabilities and limit the drag reducing performance further. Nevertheless, the evidence on permeable substrates cited above strongly suggests that the spanwise rollers are a prevalent phenomenon and will eventually appear. The onset of rollers will therefore pose a limit to the maximum achievable performance and can be used to establish a bound to this performance $a$ priori.

We consider an anisotropically permeable layer such as that depicted in Fig. 2, characterized by its thickness $h$ and its streamwise, wall-normal, and spanwise permeabilities $K_{x}, K_{y}$, and $K_{z}$, which are assumed to be along the principal directions of the permeability tensor K. Given the preliminary nature of the present analysis, we restrict ourselves to a simple model to characterize the flow within the porous substrate. We assume that the substrate is formed by a matrix of obstacles much smaller than any infinitesimal volume relevant to our problem so that homogenization can be applied on any arbitrarily small volume within the coating $[22,23]$. The permeability could decrease by increasing 


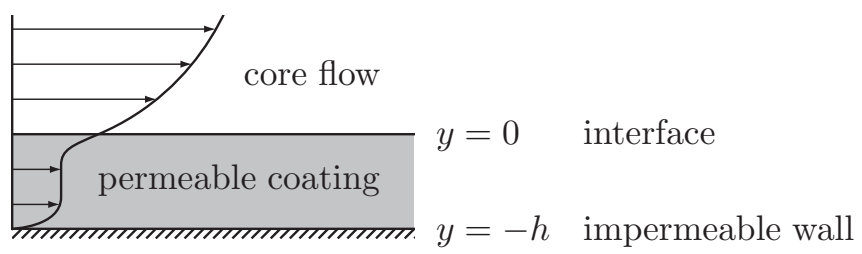

FIG. 2. Schematic representation of a permeable layer for drag reduction.

the number of obstacles, rather than their size, and vice versa. Under these conditions, the inertial terms are negligible and the resulting Stokes flow within the pores can be volume averaged to yield the Darcy equation [24]

$$
\nu \mathrm{K}^{-1} \boldsymbol{u}+\nabla p=0
$$

where $\boldsymbol{u}$ is the velocity vector; $u, v$, and $w$ are the velocity components along the principal axes; $p$ is the kinematic pressure; and $v$ is the kinematic viscosity of the fluid.

Darcy's equation is an adequate model for the flow in the core of the permeable material, but fails to represent the flow in the vicinity of impermeable walls or at the interface with an outer free flow. In order to model the flow near these interfaces, the classical approaches are the Darcy-Brinkman model and the Beavers-Joseph jump condition [25,26]. Both approaches are considered in this work and it is shown below that they lead to similar conclusions.

The paper is organised as follows. The linear drag-reducing effect is studied in Sec. II. The triggering of the drag-increasing Kelvin-Helmholtz rollers is analyzed in Sec. III, where a simplified model is proposed. Combining the results in the preceding sections, Sec. IV proposes an upper limit for the maximum drag reduction that permeable coatings could achieve. Section V summarizes the conclusions of the study.

\section{DRAG REDUCTION MECHANISM}

While for conventional smooth walls the velocity is zero at the wall, complex surfaces can yield nonzero velocities at the permeable-free-flow interface. This generates an apparent slip that can reduce drag. The theory for the underlying mechanism was proposed by Luchini et al. [4], Jiménez [6], and Luchini [7]. When the surface texture is vanishingly small with respect to the length scales in wall turbulence, the overlying turbulent eddies are comparatively very large and slow and the shear that they induce over the wall is quasihomogeneous and quasisteady sufficiently away from the wall, typically a few texture heights. Above this height, the effect of the surface can be reduced to an effective slip condition at a notional wall plane, for instance, the mean interface plane. In the streamwise and spanwise directions, we can express the slip condition in terms of the corresponding slip lengths

$$
\left.u\right|_{y=0}=\left.\ell_{x} \frac{\partial u}{\partial y}\right|_{y=0},\left.\quad w\right|_{y=0}=\left.\ell_{z} \frac{\partial w}{\partial y}\right|_{y=0},
$$

where $\ell_{x}$ and $\ell_{z}$ are the streamwise and spanwise slip lengths, as depicted in Fig. 3. In viscous units, the resulting mean streamwise slip velocity at the wall is $U_{s}^{+}=\ell_{x}^{+}$, so both slip length and slip velocity can be used interchangeably. In riblet literature, the slip lengths $\ell_{x}^{+}$and $\ell_{z}^{+}$are also referred to as protrusion heights [4,27], interpreting them as the depth below the wall where the uniform overlying shear would be extrapolated to zero, that is, where the virtual origin for the corresponding velocity profile would lie.

When the surface is anisotropic, $\ell_{x}^{+}$and $\ell_{z}^{+}$can be different. In the case of riblets, Luchini et al. [4] identified this difference as responsible for their drag-reducing capability. Jiménez [6] later 
(a)

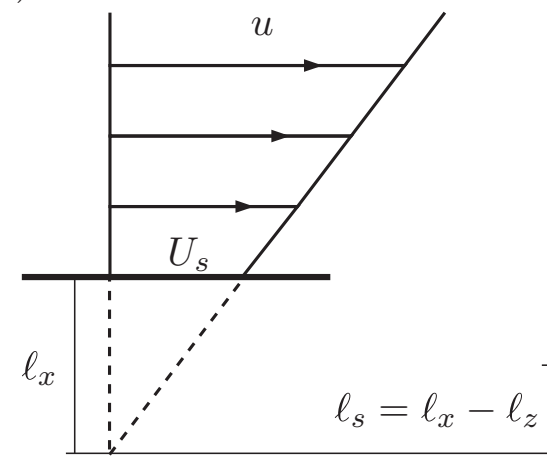

(b)
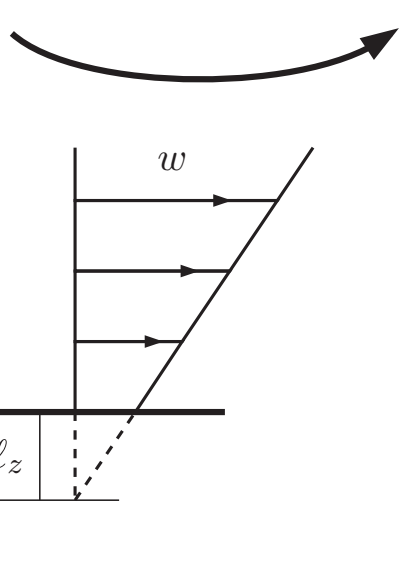

FIG. 3. Sketch of (a) streamwise and (b) spanwise slip lengths. In (b), the top arrow represents an overlying spanwise perturbation, which is perceived locally as quasisteady and quasistationary under the assumption of vanishingly small surface texture.

generalized this result to any surface producing different streamwise and spanwise slip lengths, so long as they remain small compared to the typical length scales of near-wall turbulence.

We should note that for permeable substrates the theory assumes that the characteristic size of the pores is much smaller than any other length scale in the problem and in particular the viscous length scale. A real material would have a finite pore size that, as the Reynolds number increases, would eventually not be negligible compared to the viscous length scale and the model for vanishingly small texture would stop holding. However, it is shown below that for other drag-reducing surfaces the theory holds reasonably well up to texture sizes of $O(10)$ wall units [28]. Similarly, roughness typically behaves as hydraulically smooth up to roughness sizes of three to five wall units [29], so we can expect the effect of the granularity of the surface to be negligible up to these sizes.

\section{A. Drag reduction by slip lengths}

We summarize here the relationship between $\ell_{x}, \ell_{z}$, and drag reduction, as laid out by Jiménez [6], Luchini [7], and García-Mayoral and Jiménez [30]. In the classical theory of wall turbulence, surface manipulations only modify the intercept of the logarithmic velocity profile, while the von Kármán constant $\kappa \approx 0.41$ and the wake function are unaffected [31,32]. The mean velocity profile $U$ in the logarithmic layer can be expressed as that for a smooth wall plus a shift $\Delta U$,

$$
U^{+}=\kappa^{-1} \log y^{+}+B^{+}+\Delta U^{+},
$$

where $B^{+}$is the near-wall intercept for a smooth wall and the plus superscript indicates viscous-unit scaling. The shift of the logarithmic velocity profile can be directly connected to variations in skin friction. For a constant freestream velocity and boundary layer thickness and assuming small changes of $\Delta U^{+}$and the friction coefficient $c_{f}$, Luchini [7], Spalart and McLean [33], and García-Mayoral and Jiménez [30] showed that expression (3) leads to

$$
\frac{\Delta c_{f}}{c_{f_{0}}} \approx \frac{-\Delta U^{+}}{\left(2 c_{f_{0}}\right)^{-1 / 2}+(2 \kappa)^{-1}},
$$

where $c_{f_{0}}$ is the skin friction for the reference smooth wall. If $\Delta U^{+}$is positive the logarithmic profile is shifted upward and friction is reduced, and vice versa if negative. In turn, $\Delta U^{+}$relates to the slip length induced at the surface. In the limit of vanishingly small surface manipulations, $\Delta U^{+}$satisfies

$$
\Delta U^{+}=\mu_{0}\left(\ell_{x}^{+}-\ell_{z}^{+}\right)=\mu_{0} \ell_{s}^{+},
$$


where $\mu_{0} \approx 0.66-0.785$ is a universal constant [30,34]. This concept was proposed by Luchini et al. [4] and Luchini [7] for riblets and Jiménez [6] found that Eq. (5) holds for any vanishingly small surface manipulation. Intuitively, if the crossflow is more impeded than the streamwise flow, the motion induced by quasistreamwise vortices is hindered, so they are pushed away from the notional wall perceived by the mean streamwise flow, as sketched in Fig. 3. This reduces the entrainment of momentum from layers farther away from the wall, reducing the shear at the wall and thus the skin friction. Min and Kim [35] showed that the presence of streamwise slip attenuates turbulent fluctuations, while the spanwise slip has the opposite effect, intensifying turbulence fluctuations; this had been done previously for riblets by Choi et al. [36]. Busse and Sandham [28] conducted simulations for a wide range of slip lengths and their results are consistent with Eq. (5) up to slip lengths less than or approximately equal to five wall units.

Equations (4) and (5) provide an estimate for the drag reduction $D R$ in the range of validity of small slip lengths. For typical flows at friction Reynolds numbers $\operatorname{Re}_{\tau} \approx 1000-10000$, the friction coefficient is $c_{f_{0}} \approx 0.006-0.0025$ and the drag reduction produced by a given $\ell_{x}^{+}$and $\ell_{z}^{+}$pair is

$$
D R \approx-\frac{\Delta \tau}{\tau_{0}} \approx-\frac{\Delta c_{f}}{c_{f_{0}}} \approx \frac{\mu_{0}}{\left(2 c_{f_{0}}\right)^{-1 / 2}+(2 \kappa)^{-1}} \ell_{s}^{+} \approx 0.05\left(\ell_{x}^{+}-\ell_{z}^{+}\right) .
$$

Note that Eq. (6) is constructed using Eq. (5), which holds only for small surface manipulations. For high values of $\ell_{s}^{+}$, Eq. (5), and consequently Eq. (6), would cease to hold as other mechanisms set in. In the seal fur experiments of Itoh et al. [5], for instance, $D R$ only increases up to $\ell_{s}^{+}$of order $2-4$.

\section{B. Slip lengths by porous media}

In the case of an anisotropic permeable coating such as that depicted in Fig. 2, the slip lengths can be calculated by solving the flow within the porous layer in response to an overlying shear. This is analogous to the calculation of slip lengths for superhydrophobic textures [37,38] or of protrusion heights for riblets [4]. When the porous medium is configured as a swarm of particles [25,39,40], the flow is highly connected and any section through the material would cut mostly through fluid. The macroscale shear between the fluid on either side of the section would in general not be negligible and can be accounted for by adding a macroscale viscous term to Eq. (1),

$$
\tilde{v} \nabla^{2} \boldsymbol{u}-v \mathrm{~K}^{-1} \boldsymbol{u}-\nabla p=0
$$

where the apparent viscosity $\tilde{v}$ accounts for diffusion in scales much larger than the pore size $[25,39,41]$, i.e., the large-scale diffusion that would be missed by the volume average $v \mathrm{~K}^{-1} \boldsymbol{u}$, the classical Darcy term. Equation (7) is the Darcy-Brinkman equation [24,25], which was also obtained by Taylor [39] for a matrix of infinitesimal obstacles. In general, the empirically observed $\tilde{v}$ is different from $v$, which accounts for the macroscale diffusive effects acting less efficiently than in the absence of obstacles.

If the material configuration is better represented as a matrix of microducts, rather than a swarm of obstacles, large-scale diffusion is essentially impeded and the Brinkman term should be omitted, recovering Eq. (1). At interfaces, a discontinuity in the macroscale velocity is empirically observed, which can be represented by a jump condition. For these configurations, here we use the widespread jump condition proposed by Beavers and Joseph [26], which along the streamwise direction reads

$$
\left.\frac{d U}{d y}\right|_{s}=\frac{\alpha_{\mathrm{BJ}}}{\sqrt{K_{x}}}\left(U_{s}-U_{D}\right),
$$

where $U_{D}$ is the velocity inside the coating, produced by the Darcy term, $\alpha_{\mathrm{BJ}}$ is an empirical coefficient, which depends on the interface and the permeable material, and the subindex $s$ denotes variables right above the interface.

Let us consider the flow within the coating driven by an overlying uniform streamwise shear $S_{x}$ alone. The spanwise and wall-normal velocities are zero and the pressure is homogeneous. Since 
the flow is assumed to be shear driven, $U_{D} \approx 0$ and Eq. (8) in viscous units becomes

$$
S_{x}^{+} \approx \frac{\alpha_{\mathrm{BJ}}}{\sqrt{K_{x}^{+}}} U_{s}^{+} .
$$

This equation and its analog along the spanwise direction lead to estimations of the slip lengths based on the Beavers-Joseph jump condition

$$
\ell_{x}^{+} \approx \frac{\sqrt{K_{x}^{+}}}{\alpha_{\mathrm{BJ}}}, \quad \ell_{z}^{+} \approx \frac{\sqrt{K_{z}^{+}}}{\alpha_{\mathrm{BJ}}} .
$$

Let us now address swarm-of-obstacles configurations, described by Eq. (7). Again, the pressure terms are zero and the flow is driven by the overlying uniform streamwise shear $S_{x}$. The streamwise component of Eq. (7) becomes then

$$
\frac{\partial^{2} u}{\partial y^{2}}-\frac{v}{\tilde{v}} \frac{1}{K_{x}} u=0
$$

which has solutions of the form $u \propto \exp \left(y / \sqrt{K_{x} \tilde{v} / v}\right)$. Boundary conditions can be obtained by imposing no slip at the bottom boundary, $y=-h$, and continuity of the tangential shear stress at the interface with the overlying flow, $y=0$. The resulting flow depends linearly on $S_{x}$, and the relationship between $u$ and $\partial u / \partial y$ at $y=0$ gives an $S_{x}$-independent slip length, which expressed in wall units is

$$
\ell_{x}^{+}=\sqrt{v / \tilde{v}} \sqrt{K_{x}^{+}} \tanh \left(\frac{h^{+}}{\sqrt{\tilde{v} / v} \sqrt{K_{x}^{+}}}\right)
$$

An analogous expression can similarly be derived for the spanwise slip

$$
\ell_{z}^{+}=\sqrt{v / \tilde{v}} \sqrt{K_{z}^{+}} \tanh \left(\frac{h^{+}}{\sqrt{\tilde{v} / v} \sqrt{K_{z}^{+}}}\right)
$$

Equations (10) and (12) are in agreement with the experimental measurements of Suga et al. [42], who found streamwise slip lengths of $O\left(\sqrt{K_{x}^{+}}\right)$for $\ell_{x}^{+} \lesssim 3$.

An expression for $D R$ can be obtained by introducing the estimates of Eqs. (12) and (13) into Eq. (6). As we are interested in obtaining drag reduction from a positive effective slip $\ell_{s}^{+}=$ $\ell_{x}^{+}-\ell_{z}^{+}>0$, we focus on drag reducing configurations with $\ell_{x}^{+}>\ell_{z}^{+}$and thus $K_{x}^{+}>K_{z}^{+}$. With this constraint, depending on the relative value of $h$, we can distinguish three regimes. For small values of substrate thickness $h^{+} \lesssim \sqrt{K_{z}^{+}}$, both slips tend to the same value $\ell_{x}^{+} \approx \ell_{z}^{+} \approx h^{+}$and therefore $\ell_{s}^{+} \approx 0$, resulting in no drag reduction $D R \approx 0$. For intermediate substrate thickness $\sqrt{K_{x}^{+}} \gtrsim h^{+} \gtrsim \sqrt{K_{z}^{+}}$, a positive drag reduction is obtained since $\ell_{x}^{+} \approx h^{+}$is greater than $\ell_{z}^{+} \approx \sqrt{K_{z}^{+}}$, giving as a result $D R \propto\left(h^{+}-\sqrt{K_{z}^{+}}\right)$. Finally, if $h^{+} \gtrsim \sqrt{K_{x}^{+}}$the slip lengths are $\ell_{x}^{+} \propto \sqrt{K_{x}^{+}}$and $\ell_{z}^{+} \propto \sqrt{K_{z}^{+}}$, yielding

$$
D R \approx 0.05 \sqrt{\frac{\nu}{\tilde{v}}}\left(\sqrt{K_{x}^{+}}-\sqrt{K_{z}^{+}}\right) .
$$

Of the three regimes, the latter is the most advantageous, as it gives the highest performance for a given anisotropic material with a set $\mathrm{K}$. We can conclude that, provided that the substrate has a sufficient depth $h^{+} \gtrsim \sqrt{K_{x}^{+}}$, an optimal design should have high anisotropy in order to maximize $K_{x}^{+}$while keeping $\widetilde{K_{z}^{+}}$as low as possible.

The Beavers-Joseph model leads to conclusions similar to those from the Brinkman model above. Using Eqs. (6) and (10) yields

$$
D R \approx 0.05 \frac{1}{\alpha_{\mathrm{BJ}}}\left(\sqrt{K_{x}^{+}}-\sqrt{K_{z}^{+}}\right),
$$


which closely resembles Eq. (14) and where the same influence of $\sqrt{K_{x}^{+}}-\sqrt{K_{z}^{+}}$is observed. With this model, however, $\alpha_{\mathrm{BJ}}$ takes the place of $\tilde{v} / v$ in the relationship between shear stress and velocity at the interface, with drag reduction increasing with decreasing values of $\alpha_{\mathrm{BJ}}$.

Equations (14) and (15) also allow us to identify the influence of the permeability and thickness of the substrate. The drag reduction is essentially determined by the difference between streamwise and spanwise permeabilities $\sqrt{K_{x}^{+}}-\sqrt{K_{z}^{+}}$and the wall-normal permeability does not play a significant role in this linear regime. Both models agree that the permeable material should ideally be highly anisotropic in order to obtain a large $\sqrt{K_{x}^{+}}-\sqrt{K_{z}^{+}}$and therefore high drag reduction. These models also show that the connectivity of the microstructure of the substrate, condensed in either $\tilde{v}$ or $\alpha_{\mathrm{BJ}}$, also plays an important role. Materials with high connectivity will allow larger slip at the interface and therefore have a positive effect on $D R$. We must however note that expressions (14) and (15) only provide an estimate of the order of magnitude of the drag reduction and that a more refined characterization of the permeable substrate would be required for more precise estimates. The main source of uncertainty in Eqs. (14) and (15) are the estimates for $\tilde{v} / v$ or $\alpha_{\mathrm{BJ}}$, which encapsulate the interaction between fluid and porous material at the interface. Zampogna and Bottaro [22] and Lācis and Bagheri [23] have recently proposed homogenization techniques to predict this interaction accurately.

As we can see, using either model results in expressions for the slip lengths that closely resemble each other, when deep coatings are assumed. The coefficients $\alpha_{\mathrm{BJ}}$ and $\sqrt{\tilde{v} / v}$ play the same role, as proposed by Neale and Nader [43], which allows us to write Eqs. (14) and (15) in the form

$$
D R \approx 0.05 \xi\left(\sqrt{K_{x}^{+}}-\sqrt{K_{z}^{+}}\right)
$$

where $\xi$ is either $\sqrt{v / \tilde{v}}$ or $\alpha_{\mathrm{BJ}}^{-1}$.

This result provides an expression for the order of magnitude of drag reduction obtained by permeable coatings. The above derivation neglects the effect of pressure within the substrate, which is negligible in most industrial applications. For example, in order to produce drag reduction of $O(10 \%)$, the permeability of the material would be $\sqrt{K_{x}^{+}} \approx 2-3$, which would require a thickness $h^{+} \sim O(10)$. In industrial ducts and pipelines, for instance, the Reynolds number is $10^{5}-10^{7}$ and the above thickness would correspond to $h \sim O(100 \mu \mathrm{m})$, resulting in an additional cross section of $O(1 \%)$. The additional drag caused by the pressure drop acting in this increased cross section would then be $O(1 \%)$, much smaller than the $D R$ caused by the slip effect. Moreover, this pressure drop would create additional Darcy flow within the coating, resulting in increased flow rate not only within the coating, but also across the whole section. This would mitigate the deleterious effect and could even negate it, depending on the coating parameters. In turn, in external flows, like aeroplane fuselages, the pressure gradient is generally milder and therefore its effect on the substrate would not contribute significantly to drag. The coating thickness would also be of order of microns in this case and the shape, and hence the form drag, would not change noticeably.

\section{LIMITING MECHANISM FOR DRAG REDUCTION}

Just like in the classical studies of Jiménez [6] and Luchini [7], the model presented in Sec. II predicts a drag reduction ever increasing with the surface permeability, scaled in viscous units. Let us take a given substrate configuration $\left(K_{x}, K_{z}, K_{y}, h\right)$, yielding an effective slip $\ell_{s}>0$. By simply increasing the friction velocity of the flow, the viscous length scale would decrease and $\sqrt{K_{x}^{+}}, \sqrt{K_{z}^{+}}$, and $h^{+}$would increase by the same proportion, with the corresponding increase in $D R$, as given by Eq. (16). This is obviously not the case, because the theory and the resulting models cease to hold once the texture size, in viscous units, reaches a certain value. The theory developed by Luchini et al. [4], Luchini [7], and Jiménez [6] assumes slip lengths much smaller than one viscous unit, or in our case $\sqrt{K_{x}^{+}} \ll 1$. The linear behavior is nevertheless observed to hold for $\sqrt{K_{x}^{+}} \lesssim 5$, but even beyond this value the drag reduction keeps increasing monotonically with $\sqrt{K_{x}^{+}}$[28]. In experience, 
however, other mechanisms, different from the slip effect and dependent on the type of surface, can be expected to set in at some $\sqrt{K_{x}^{+}}$and limit the drag reducing capability of the surface.

In an attempt to establish some limit for the linear behavior of Eq. (16), we propose a model to predict the onset of the Kelvin-Helmholtz-like rollers discussed in the Introduction. While other deleterious effects may appear at smaller $\sqrt{K_{x}^{+}}$, the evidence on permeable substrates strongly suggests that such rollers will eventually appear [11-14], causing additional Reynolds stresses that will degrade drag $[8,11,12]$. Other phenomena, limiting the performance further, cannot be ruled out, but the present model gives an order-of-magnitude limit that could not be exceeded by permeable surfaces. This can therefore be interpreted as an upper bound for the maximum drag reduction achievable.

\section{A. Model from linear instability}

To capture the onset of Kelvin-Helmholtz-like rollers, we propose a model based on the linear stability analysis of the mean flow in a turbulent channel with symmetric permeable walls. Mean-flow analysis has been shown to adequate capture instabilities, and in particular Kelvin-Helmholtz rollers, in flows with a fluctuating turbulent component $[44,45]$ and has been extensively used in turbulent flows over complex surfaces $[8,11,19,46]$. Here we follow closely the methodology of Jiménez et al. [11] and García-Mayoral and Jiménez [8].

Kelvin-Helmholtz instabilities are essentially inviscid and linear and can therefore be captured by the equations for linearised inviscid perturbations [11,19], with the fully viscous analysis showing no fundamental difference in the results [11,47]. Therefore, we restrict our analysis to the inviscid case. We conduct the analysis on the flow outside the permeable substrate and the influence of the substrate appears as a boundary condition at the interface, provided by the analytical solution of the underlying flow, detailed below.

We seek wavelike solutions for the velocity and pressure perturbations, of the form $f=$ $\hat{f} \exp [i(\alpha x+\beta z-\omega t)]$, which permits a modal analysis. The resulting expression is Rayleigh's equation [48]

$$
(U-c)\left(\partial_{y y}-k^{2}\right) \hat{v}-U^{\prime \prime} \hat{v}=0,
$$

where $U$ is the base flow, $\alpha$ and $\beta$ are the wave numbers in the streamwise and spanwise directions, respectively, $\hat{v}$ is the corresponding perturbation mode of the wall-normal velocity, $k^{2}=\alpha^{2}+\beta^{2}$, $\omega$ is the complex frequency, $c$ is the complex phase velocity defined as $\omega=\alpha c$, and the primes indicate derivatives with respect to $y$.

The difference between the present analysis and a smooth-wall case lies in the presence of the permeable substrate, which imposes an impedancelike boundary condition on the core flow $[8,11,49]$. To derive the boundary condition, we focus on the response of the flow within the permeable medium to the overlying pressure fluctuations $\left.\hat{p}\right|_{y=0^{+}}$. Note that this flow regime is entirely different from that discussed in Sec. II B, which was driven by shear. Indeed, since Kelvin-Helmholtz is an inviscid instability, the perturbation field of the overlying flow is now assumed to be inviscid, so it cannot exert any shear on the flow below, which is therefore free to slip with respect to the external velocity.

Let us now consider the flow within the substrate on the bottom wall of the channel, which extends from $y=-h$ to $y=0$. The flow is described by Eq. (7). The Brinkman diffusive term $\tilde{v} \nabla^{2} u$ is typically negligible except in thin layers, of thickness of order $\sim \sqrt{K_{x}}$, where the flow transitions from the Darcy solution to the no-slip boundary value, as it does in boundary layers for external flows. As we consider deep coatings $h \gg \sqrt{K_{x}}$, the Brinkman layer at $y=-h$ is far from the flow near $y=0$ and does therefore not have an influence on it. At the same time, at $y=0$ there is free slip with the inviscid perturbation flow above and no Brinkman layer forms. In the absence of Brinkman layers, we neglect the effect of the Brinkman term and Eq. (7) becomes the classical Darcy equation (1). Combined with incompressibility, it leads to a Poisson equation for $\hat{p}$. Assuming impermeability at $y=-h$, the solution is a function of the value of $\hat{p}$ at $y=0$,

$$
\hat{p}=\hat{p}_{0}\left[\tanh \left(\tilde{\alpha} h \Phi_{x y}\right) \sinh \left(\tilde{\alpha} y \Phi_{x y}\right)+\sinh \left(\tilde{\alpha} y \Phi_{x y}\right)\right],
$$



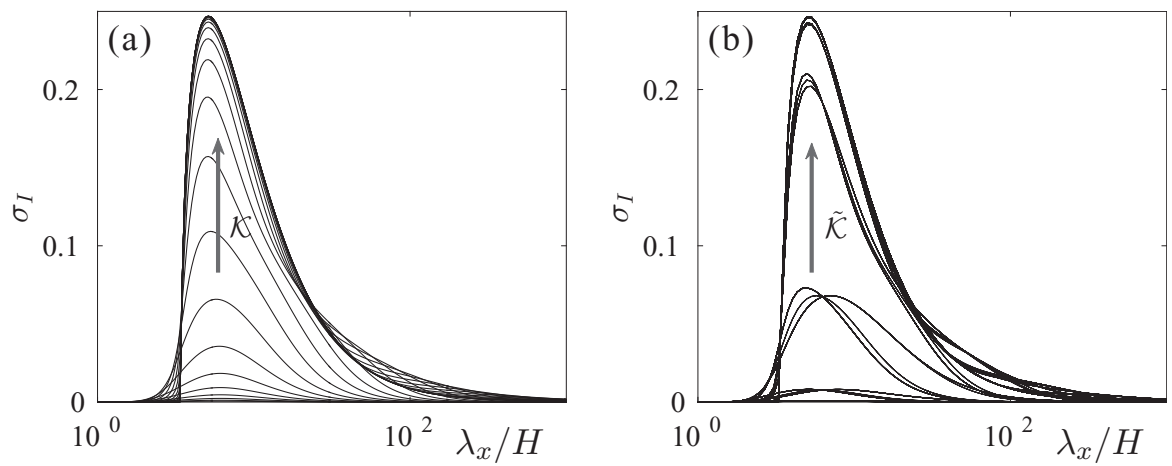

FIG. 4. Growth rate $\sigma_{I}=\operatorname{Im}(\sigma)$ of the most amplified modes given by (21). (a) Isotropic case with $\Phi_{x y}=1$ and $h / H=1$. Curves are shown for $\left(\sqrt{K_{x} K_{y}} / H^{2}\right)(U H / v)=10^{[-2(0.4) 6]}$. (b) Anisotropic case obtained using all possible combinations of $h / H=10^{[-1,0,1]}$ and $\Phi_{x y}=10^{[-3(1) 3]}$. Curves are shown for $\tilde{\mathcal{K}}=10^{[-2(1) 6]}$.

where $\Phi_{x y}=\sqrt{K_{x} / K_{y}}$ is the streamwise-to-wall-normal anisotropy ratio, $\tilde{\alpha}^{2}=\alpha^{2}+\beta^{2} K_{z} / K_{x}$, and the subscript 0 indicates magnitudes at the interface $y=0$. This solution can be introduced in the Darcy equation for the wall-normal velocity, giving

$$
\hat{v}_{0}=-\tilde{\alpha}\left[v^{-1} \sqrt{K_{x} K_{y}} \tanh \left(\tilde{\alpha} h \Phi_{x y}\right)\right] \hat{p}_{0} .
$$

Equation (19) can be used as an impedance boundary condition in the stability analysis of the overlying flow. On the opposite side of the channel, an analogous impedance condition can be obtained, resulting in the same form of Eq. (19), but reversed in sign.

As in the work of Jiménez et al. [11] and Tilton and Cortelezzi [50], Squire's transformation [51] reduces the problem of Eqs. (17)-(19) to an equivalent spanwise-homogeneous, two-dimensional problem with $\alpha_{2 \mathrm{D}}=\tilde{\alpha}, \beta_{2 \mathrm{D}}=0$, and modified permeabilities. The equivalent problem has lower permeabilities than the spanwise-homogeneous, two-dimensional problem with $\alpha=\alpha_{2 \mathrm{D}}$ and $\beta=0$ and, as shown below, reducing the permeabilities has a stabilizing effect. Consequently, we will only consider solutions with $\beta=0$, as for each $\alpha$ they are the most amplified. Note that this is in agreement with the observed Kelvin-Helmholtz structures being predominantly spanwise coherent $[8,11,18,21]$.

\section{B. Results for a piecewise-linear mean velocity profile}

Before turning our attention to a quantitative analysis using turbulent mean profiles as base flows, it is useful to study a piecewise-linear one

$$
U(y)= \begin{cases}U_{\infty} y / H & \text { for } y<H \\ U_{\infty} & \text { for } y \geqslant H\end{cases}
$$

For this profile the solution is algebraic and the basic mechanisms are more easily understood. Note that the base flow has $U^{\prime \prime}=0$ everywhere except at $y=H$, where it becomes singular. Equations (17) and (19) lead then to a second-order equation for the complex phase velocity $c$,

$$
-2 \mathcal{K} \sigma^{2}+\left[-2 i+\mathcal{K}\left(1+2 \alpha^{\prime}-e^{-2 \alpha^{\prime}}\right)\right] \sigma+(\mathcal{K}-i)\left(1-2 \alpha^{\prime}-e^{-2 \alpha^{\prime}}\right)=0,
$$

where $\alpha^{\prime}=\alpha H, \sigma=\alpha^{\prime} c / U_{\infty}$, and

$$
\mathcal{K}=\frac{\sqrt{K_{x} K_{y}}}{H^{2}}\left(\frac{U_{\infty} H}{v}\right) \tanh \left(\alpha^{\prime} \frac{h}{H} \Phi_{x y}\right)
$$

The results of (21) as a function of $\mathcal{K}$ are shown in Fig. 4(a). The limit $\mathcal{K} \gg 1$ provides a physical interpretation of the nature of the instability. In this limit, the boundary condition (19) is equivalent to 
$p(0)=0$, which can be reduced to $\partial_{y} \hat{v}(0)=0$, and enforces symmetry on the perturbation flow. This gives the same solution as extending the base profile antisymmetrically about $y=0$. The problem becomes then that of the instability of a shear layer, whose solutions are the Kelvin-Helmholtz unstable waves of a free shear layer. In the opposite limit $\mathcal{K} \ll 1$, the neutrally stable solution of smooth impermeable walls is asymptotically approached. The intermediate values of $\mathcal{K}$ connect the Kelvin-Helmholtz solution with the stable solution for the impermeable case.

Expression (22) depends not only on the properties of the porous coating, but also on the reduced spectral wave number $\alpha^{\prime}$, which is not a physical property of the permeable layer but part of the solution. In an attempt to remove the dependence on the flow we propose the empirical fit

$$
\tilde{\mathcal{K}}=\frac{\sqrt{K_{x} K_{y}}}{H^{2}}\left(\frac{U_{\infty} H}{v}\right) \tanh \left(\frac{h}{H} \Phi_{x y}\right)
$$

Figure 4(b) shows results, as a function of $\tilde{\mathcal{K}}$, for several combinations of $K_{x}, K_{y}$, and $h$. For the same values of $\tilde{\mathcal{K}}$, solutions for different coatings agree well, except perhaps for low values of $\tilde{\mathcal{K}}$, for which the instability is not fully developed. Other than for those low values, the parameter $\tilde{\mathcal{K}}$ encompasses the combined influence of $K_{x}, K_{y}$, and $h$. Note that, while the amplification is determined by $\tilde{\mathcal{K}}$, the most amplified wavelength does not scale with the characteristic permeability length scale, $\sqrt{K_{x}}$ or $\sqrt{K_{y}}$. As in the work of García-Mayoral and Jiménez [8], the wavelength scales with the height $y=H$ of the singularity in $U^{\prime \prime}$, that is, it is determined by the shape of the base flow.

\section{Results for turbulent mean velocity profile}

Although the analysis on the piecewise-linear velocity profile provides qualitative information on the nature of the instability, quantitative results require more realistic profiles. We use approximate turbulent mean profiles as in the work of Jiménez et al. [11], Dupont et al. [46], and García-Mayoral and Jiménez [8]. In particular, we use the profiles of Cess [52], which have previously been used for flow stability analysis by Reynolds and Tiederman [53] and more recently by del Álamo and Jiménez [54]. In contrast with the linear profile used in the preceding section, the stability problem (17), with the boundary condition (19), no longer leads to an algebraic expression and the full generalized eigenvalue problem must be discretized and solved numerically. For every Fourier mode $\alpha$ the wall-normal direction is discretized using Chebyshev polynomials with 256-1536 collocation points, depending on the Reynolds number, which provide a resolution at the wall $\Delta y^{+} \lesssim 0.01$. Obtaining insight from the solution becomes less straightforward, but some analogies can be established with the piecewise-linear results.

As in Sec. III B, we find that the length scale of the problem is determined by the shape of the $U$ profile. The energy-producing term $U^{\prime \prime}$ is larger between $y^{+}=5$ and 20, peaking near $y_{c}^{+} \approx 8$, which plays the same role as the singularity at $y=H$ for the piecewise-linear profile [8]. This height is independent of the Reynolds number when scaled in wall units and is responsible for the inner scaling of the instability observed in Fig. 5(a). The solution, portrayed in Fig. 5(a) for isotropic substrates, is qualitatively similar to the one for the piecewise-linear profile, evolving as the permeability increases from the neutral smooth-wall solution to increasingly amplified solutions and eventually reaching a limit solution for high permeabilities.

We are particularly interested in the most amplified mode for each surface configuration, as this will be the most prevalent [8,11]. This mode forms rollers turning alternatively clockwise and counterclockwise that penetrate into the porous material below $y^{+}=0$, as portrayed in Fig. 5(b).

As in Sec. III B, we aim to describe the solution using a simplified characterizing parameter. By analogy with Eq. (23) we propose

$$
\tilde{K}^{+}=\sqrt{K_{x}^{+} K_{y}^{+}} \tanh \left(\frac{h^{+}}{y_{c}^{+}} \Phi_{x y}\right)
$$



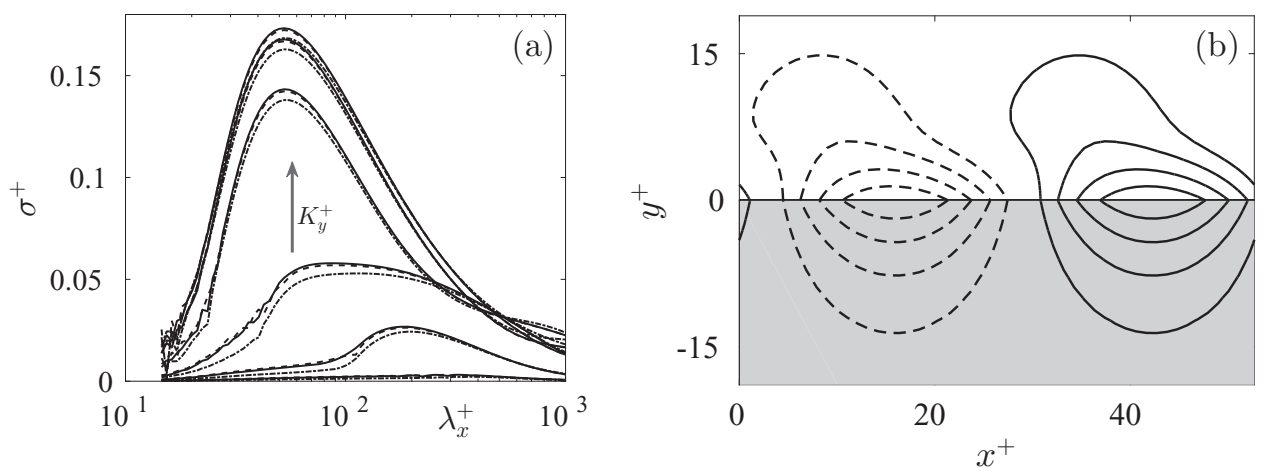

FIG. 5. (a) Growth rate $\sigma^{+}=\alpha^{+} \operatorname{Im}\left(c^{+}\right)$of the most amplified mode as a function of the longitudinal wavelength $\lambda_{x}^{+}$: -.--, $\operatorname{Re}_{\tau}=180 ;--, \operatorname{Re}_{\tau}=550 ;-, \operatorname{Re}_{\tau}=1000$. For the isotropic case $\Phi_{x y}=1, K^{+}=$ $10^{[-0.66(0.66) 2.66]}, h^{+}=100$. (b) Stream-function contours of the mode with the highest growth rate at $\operatorname{Re}_{\tau}=550$ for fully developed instability $\tilde{K}^{+}=10^{4}$. Solid and dashed lines correspond to clockwise and counterclockwise rotation, respectively.

Figure 6(a) illustrates how scaling with this parameter results in a reasonable collapse for different $K_{x}^{+}, K_{y}^{+}$, and $h^{+}$. For high values of $\tilde{K}^{+}$there is good agreement, while for low values we observe some scatter depending on the value of $h^{+} \Phi_{x y}$. The growth rate of the most amplified mode for each set of parameters is portrayed separately in Fig. 6(b) as a function of $\tilde{K}^{+}$, showing that the effect of the modulation with $h^{+} \Phi_{x y}$ is small and only appears for low permeabilities $\tilde{K}^{+} \lesssim 5$. García-Mayoral and Jiménez [8] found a similar S-shaped curve for the relationship between $\sigma^{+}$and the characteristic length scale of riblets. In their case, the degradation of drag reduction empirically observed roughly coincided with the sharp transition between the quasineutral and the fully amplified regimes. They suggested that the model could therefore be used as an indicator for the onset of Kelvin-Helmholtz-like rollers and to obtain estimates for the riblet size, in viscous units, for which the degradation of drag would roughly set in.

In the present case, the transition between the quasineutral and fully amplified regimes occurs at $\tilde{K}^{+} \approx 5-10$. Note that, beyond $\tilde{K}^{+}=5$, the scatter for low values of $\tilde{K}^{+}$discussed above is not significant. Hence, $\Phi_{x y} h^{+}$should have little effect on the triggering of rollers. For that reason,
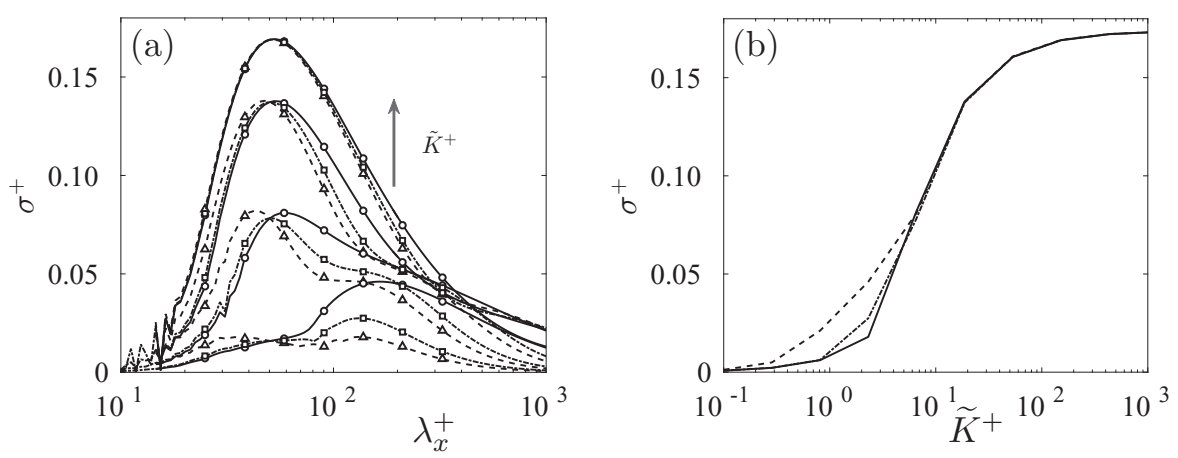

FIG. 6. (a) Growth rate $\sigma^{+}=\alpha^{+} \operatorname{Im}\left(c^{+}\right)$of the most amplified mode as a function of the longitudinal wavelength $\lambda_{x}$ : -,$\Phi_{x}=10^{-3}$ and $h^{+}=10 ;--, \Phi_{x}=10^{3}$ and $h^{+}=10 ; \Delta, \Phi_{x}=1$ and $h^{+}=1 ; \circ$, $\Phi_{x}=1$ and $h^{+}=100 ; \square, \Phi_{x}=1$ and $h^{+}=10$. Here $\tilde{K}^{+}=10^{[0.36,0.82,1.28,2.20]}$ at $\operatorname{Re}_{\tau}=550$. (b) Maximum growth rate $\sigma^{+}$as a function of the permeability $\tilde{K}^{+}$at $\operatorname{Re}_{\tau}=550:-, \Phi_{x}=10^{-3}$ and $h^{+}=1 ;--, \Phi_{x}=10^{3}$ and $h^{+}=100 ;-\cdot-, \Phi_{x}=1$ and $h^{+}=10$. 


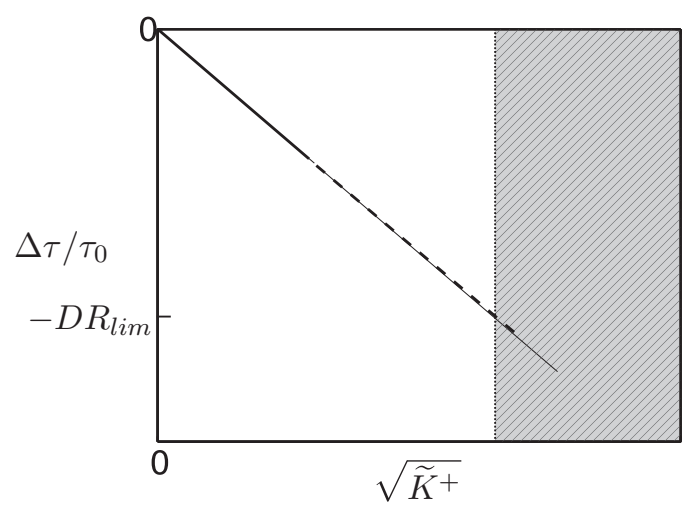

FIG. 7. Schematic summarizing the initial linear behavior of $D R=\Delta \tau / \tau_{0}$ for low permeability. The hatched area represents the region $\tilde{K}>\tilde{K}_{\mathrm{KH}}$, where the drag reducing effect is destroyed by the appearance of Kelvin-Helmholtz rollers.

the term $\tanh \left(\Phi_{x y} h^{+} / y_{c}^{+}\right)$in expression (24) can be approximated by its rapidly approached limit $\tanh \left(\Phi_{x y} h^{+} / y_{c}^{+}\right) \approx 1$, taking into account that $y_{c}^{+} \approx 8$ and that for the configurations of practical interest $\Phi_{x y} \gg 1$ and $h^{+} \gtrsim 10$. The criterion for the onset of the Kelvin-Helmholtz-like instability can then be roughly set to

$$
\tilde{K}_{\mathrm{KH}}^{+} \approx \sqrt{K_{x}^{+} K_{y}^{+}} \approx 5-10 .
$$

For flows over permeable substrates of diverse depth and permeability, it is difficult to find in the literature details of the structure of the near-wall flow. Breugem et al. [12] reported the appearance of Kelvin-Helmholtz rollers at $\tilde{K}^{+} \approx 80$. Zampogna et al. [15], on their permeable layer model for canopy flow, also observe rollers at $\tilde{K}^{+} \approx 300$. It is worth noting that, although Rosti et al. [13] did not observe Kelvin-Helmholtz rollers directly in their simulations, with $\tilde{K}^{+}<1$, they observed a weak Kelvin-Helmholtz signal in their velocity correlations. This is in agreement with the very low amplification that our model predicts for low $\tilde{K}^{+}$.

In any event, the appearance of rollers for $\tilde{K}^{+} \gtrsim \tilde{K}_{\mathrm{KH}}^{+}$will degrade the linear performance assumed in Sec. II, as the rollers introduce additional Reynolds stresses [8]. In the absence of earlier degrading mechanisms, the maximum drag reduction would occur for $\tilde{K}^{+}=\tilde{K}_{\mathrm{KH}}^{+}$, so $\tilde{K}_{\mathrm{KH}}^{+}$can be taken as an upper bound for the limit of the drag-decreasing regime.

\section{LIMIT TO DRAG REDUCTION BY PERMEABLE COATINGS}

In Sec. II we analyzed the drag reduction for substrates of vanishing permeability. For these substrates, the slip lengths can be estimated as a function of the permeability, resulting in Eq. (16), which connects $D R$ with the properties of a particular permeable substrate. Within the limits of the vanishingly small assumption, Eq. (16) denotes an ever-increasing $D R$ with $\sqrt{K_{x}^{+}}$. This result is consistent with numerical experiments on walls with anisotropic slip lengths $[3,28,35]$. However, this behavior will eventually fail for large permeabilities, once the assumptions in the model break down or additional mechanisms set in. To bound the range of validity of the model, in Sec. III we investigated the appearance of Kelvin-Helmholtz-like rollers, which are a common feature in flows over porous materials $[11,12]$. The appearance of these rollers results in enhanced mixing, increasing drag and posing a limitation to the aforementioned linear behavior. These two concepts are depicted together in Fig. 7, which shows the initial linear behavior for low permeabilities as well as the limit to drag reduction $D R_{\lim }$ for high permeabilities, due to the Kelvin-Helmholtz instability. Note that $D R_{\text {lim }}$ must only be considered as an upper limit estimate, since in the intermediate 


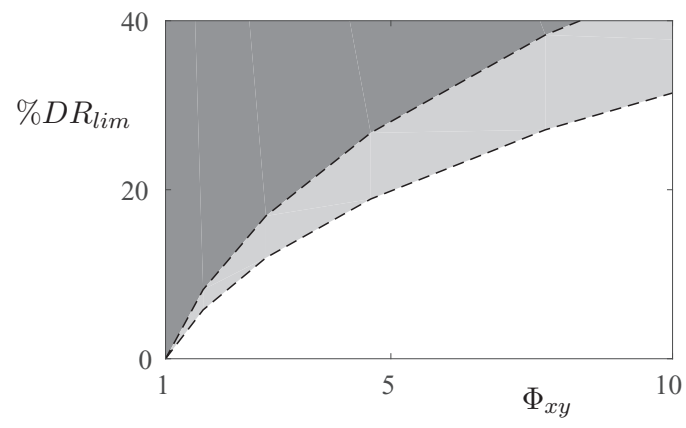

FIG. 8. Limit for the maximum drag reduction achievable as a function of the anisotropy ratio of the permeable layer $\Phi_{x y}$. The dashed lines denote Eq. (26) for $\tilde{K}_{\mathrm{KH}}^{+}=5$ and 10 , and $\xi=1$. The light gray shaded region indicates the range for which Kelvin-Helmholtz rollers are expected to appear. The dark gray shaded region indicates the range for which the Kelvin-Helmholtz rollers would be fully developed.

range of permeabilities beyond $\sqrt{K_{x}^{+}} \ll 1$ we cannot rule out additional mechanisms that may degrade drag further. For riblets, the analogous linear behavior roughly extends until the onset of the Kelvin-Helmholtz mechanism [8], although this may not be the case for the present porous coatings, as other degrading phenomena could appear before reaching $\tilde{K}_{\mathrm{KH}}^{+}$.

Section II shows that in order to obtain high drag reduction one would need to maximize the difference $\sqrt{K_{x}^{+}}>\sqrt{K_{z}^{+}}$, while in order to delay the appearance of the drag degrading spanwise rollers the product of permeabilities needs to stay below a threshold $\sqrt{K_{x}^{+} K_{y}^{+}}<\tilde{K}_{K H}^{+}$, as shown in Sec. III. A high value of $\xi$, corresponding to substrates with sparse internal structure, is also desirable, noticing that previous research appears to agree that $\xi$ tends to 1 for very sparse porous matrices [40]. These results can be combined to obtain an estimate for the limit to drag reduction produced by different substrates. Let us consider a permeable material with a preferential permeability $K_{x}^{+}>$ $K_{y}^{+}=K_{z}^{+}$. The resulting anisotropy ratio $\Phi_{x y}=\sqrt{K_{x} / K_{y}}=\sqrt{K_{x} / K_{z}}$ can be used in Eqs. (16) and (25) to obtain an expression for the order of magnitude of the upper limit for drag reduction

$$
D R_{\lim } \approx 0.05 \xi\left(1-\Phi_{x y}^{-1}\right) \sqrt{\Phi_{x y} \tilde{K}_{\mathrm{KH}}^{+}} .
$$

Figure 8 portrays an example of the resulting $\Phi_{x y}-D R$ curves for both $\tilde{K}_{\mathrm{KH}}^{+}=5$ and 10 , in the limit of $\xi=1$. The region enclosed by the two curves represents the range for which Kelvin-Helmholtz rollers can be expected to appear and delimits the range of realizable values for $D R$. For an anisotropy ratio of order $\Phi_{x y} \approx 5-10$ the maximum drag reduction achievable would be below 20-30\%. Note, however, that for substrates that impede diffusion the drag reduction capability can be substantially smaller. For the substrate of [23], for instance, $\xi \approx 0.25$, which would yield a drag reduction of no more than $5-7 \%$. Thus, we can only estimate that $D R_{\lim }=O(10 \%)$ with an anisotropy of order $\Phi_{x y} \approx 5-10$.

\section{CONCLUSION}

In the present work we have proposed a simplified model to estimate the drag reduction properties of anisotropically permeable coatings. For small permeabilities, permeable substrates can potentially reduce skin friction if the surface obstructs more the spanwise than the streamwise flow, as riblets and some other drag reducing surfaces do. Using simplified models, a relationship between drag reduction, slip length at the interface, and the properties of the porous material is established. This results in the drag reduction being approximately proportional to the difference between the streamwise and spanwise permeabilities, provided that the coating is sufficiently deep, $h^{+} \gtrsim \sqrt{K_{x}^{+}}>$ $\sqrt{K_{z}^{+}}$. 
We have also investigated a limit to the drag-reducing capabilities of these surfaces, given by the triggering of Kelvin-Helmholtz-like rollers over the surface. These are common features of turbulent flows over permeable substrates and other complex surfaces and are responsible for increased momentum transfer that degrades drag. Their appearance will limit the drag reduction performance, which, in the absence of degrading mechanisms, would continuously improve as the permeability of the surface, measured in wall units, increases. This can be used to set an upper bound for the performance of the surfaces under consideration. A model derived from inviscid linear stability analysis indicates that the critical parameters for the onset of rollers scale in viscous units. The appearance of rollers is estimated to trigger for $\sqrt{K_{x}^{+} K_{y}^{+}} \gtrsim \tilde{K}_{\mathrm{KH}}^{+} \approx 5-10$. This implies that the anisotropically permeable substrates considered could yield drag reductions of $O(10 \%)$, for anisotropy ratios of $\sqrt{K_{x}^{+} / K_{y}^{+}}=\sqrt{K_{x}^{+} / K_{z}^{+}} \approx 5-10$. This preliminary figure is promising, but requires further study to delimit more precisely the potential of these surfaces.

\section{ACKNOWLEDGMENTS}

N.A-E. was supported by the Engineering and Physical Sciences Research Council (Grant No. EP/M506485/1). The authors wish to express their gratitude to J. Jiménez for the inspiring seminal discussions on fibrous riblets and his thoughtful comments. We also express our gratitude to S. Bagheri and the reviewers for the fruitful discussion on the modeling of permeable media, which significantly enriched the contents of this paper.

[1] M. J. Walsh and A. M. Lindemann, Optimization and application of riblets for turbulent drag reduction, Proceedings of the 22nd Aerospace Sciences Meeting (AIAA, Reston, VA, 1984).

[2] J. P. Rothstein, Slip on superhydrophobic surfaces, Annu. Rev. Fluid Mech. 42, 89 (2010).

[3] S. Hahn, J. Je, and H. Choi, Direct numerical simulation of turbulent channel flow with permeable walls, J. Fluid Mech. 450, 259 (2002).

[4] P. Luchini, F. Manzo, and A. Pozzi, Resistance of a grooved surface to parallel flow and cross-flow, J. Fluid Mech. 228, 87 (1991).

[5] M. Itoh, S. Tamano, R. Iguchi, K. Yokota, N. Akino, R. Hino, and S. Kubo, Turbulent drag reduction by the seal fur surface, Phys. Fluids 6, 065102 (2006).

[6] J. Jiménez, On the structure and control of near wall turbulence, Phys. Fluids 6, 944 (1994).

[7] P. Luchini, Reducing the turbulent skin friction, European Congress on Computational Methods in Applied Sciences ECCOMAS CFD (Springer, Berlin, 1996), pp. 466-470.

[8] R. García-Mayoral and J. Jiménez, Hydrodynamic stability and breakdown of the viscous regime over riblets, J. Fluid Mech. 678, 317 (2011).

[9] J. Seo, R. Garcia-Mayoral, and A. Mani, Pressure fluctuations and interfacial robustness in turbulent flows over superhydrophobic surfaces, J. Fluid Mech. 783, 448 (2015).

[10] R. García-Mayoral, J. Seo, and A. Mani, Dynamics of gas-liquid interfaces in turbulent flows over superhydrophobic surfaces, in Studying Turbulence Using Numerical Simulation Databases-XV, Proceedings of the 2014 Summer Program (Stanford University Press, Stanford, 2014), pp. 295-304.

[11] J. Jiménez, M. Uhlmann, A. Pinelli, and G. Kawahara, Turbulent shear flow over active and passive porous surfaces, J. Fluid Mech. 442, 89 (2001).

[12] W. P. Breugem, B. J. Boersma, and R. E. Uittenbogaard, The influence of wall permeability on turbulent channel flow, J. Fluid Mech. 562, 35 (2006).

[13] M. E. Rosti, L. Cortelezzi, and M. Quadrio, Direct numerical simulation of turbulent channel flow over porous walls, J. Fluid Mech. 784, 396 (2015).

[14] Y. Kuwata and K. Suga, Lattice Boltzmann direct numerical simulation of interface turbulence over porous and rough walls, Int. J. Heat Fluid Flow 61, 145 (2016). 
[15] G. A. Zampogna, F. Pluvinage, A. Kourta, and A. Bottaro, Instability of canopy flows, Water Resour. Res. 52, 5421 (2016).

[16] P. Orlandi and J. Jiménez, On the generation of turbulent wall friction, Phys. Fluids 6, 634 (1994).

[17] P. Orlandi, The importance of wall-normal Reynolds stress in turbulent rough channel flows, Phys. Fluids 25, 110813 (2013).

[18] J. Finnigan, Turbulence in plant canopies, Annu. Rev. Fluid Mech. 32, 519 (2000).

[19] C. Py, E. De Langre, and B. Moulia, A frequency lock-in mechanism in the interaction between wind and crop canopies, J. Fluid Mech. 568, 425 (2006).

[20] O. Coceal, A. Dobre, T. G. Thomas, and S. E. Belcher, Structure of turbulent flow over regular arrays of cubical roughness, J. Fluid Mech. 589, 375 (2007).

[21] M. Ghisalberti, Obstructed shear flows: Similarities across systems and scales, J. Fluid Mech. 641, 51 (2009).

[22] G. A. Zampogna and A. Bottaro, Fluid flow over and through a regular bundle of rigid fibres, J. Fluid Mech. 792, 5 (2016).

[23] U. Lācis and S. Bagheri, A framework for computing effective boundary conditions at the interface between free fluid and a porous medium, J. Fluid Mech. 812, 866 (2017).

[24] H. Darcy, Les Fontaines Publiques de la Ville de Dijon (Dalmont, Paris, 1856).

[25] H. C. Brinkman, A calculation of the viscous force exerted by a flowing fluid on a dense swarm of particles, Appl. Sci. Res. 1, 27 (1949).

[26] G. S. Beavers and D. D. Joseph, Boundary conditions at a naturally permeable wall, J. Fluid Mech. 30, 197 (1967).

[27] D. W. Bechert and M. Bartenwerfer, The viscous flow on surfaces with longitudinal ribs, J. Fluid Mech. 206, 105 (1989).

[28] A. Busse and N. D. Sandham, Influence of an anisotropic slip-length boundary condition on turbulent channel flow, Phys. Fluids 24, 055111 (2012).

[29] J. Jiménez, Turbulent flows over rough walls, Annu. Rev. Fluid Mech. 36, 173 (2004).

[30] R. García-Mayoral and J. Jiménez, Drag reduction by riblets, Philos. Trans. R. Soc. A 369, 1412 (2011).

[31] J. Nikuradse, Laws of flow in rough pipes, NACA Report No. 1292, 1933 (unpublished).

[32] F. H. Clauser, The turbulent boundary layer, Adv. Appl. Mech 4, 1 (1956).

[33] P. R. Spalart and J. D. McLean, Drag reduction: Enticing turbulence, and then industry, Philos. Trans. R. Soc. A 369, 1556 (2011).

[34] D. W. Bechert, M. Bruse, W. Hage, J.G.T. van der Hoeven, and G. Hoppe, Experiments on drag-reducing surface and their optimization with an adjustable geometry, J. Fluid Mech. 338, 59 (1997).

[35] T. Min and J. Kim, Effects of hydrophobic surface on skin-friction drag, Phys. Fluids 16, 1 (2004).

[36] H. Choi, P. Moin, and J. Kim, Direct numerical simulation of turbulent flow over riblets, J. Fluid Mech. 255, 503 (1993).

[37] J. R. Philip, Flows satisfying mixed no-slip and no-shear conditions, Z. Angew. Math. Phys. 23, 353 (1972).

[38] C. Ybert, C. Barentin, and C. Cottin-Bizonne, Achieving large slip with superhydrophobic surfaces: Scaling laws for generic geometries, Phys. Fluids 19, 123601 (2007).

[39] G. I. Taylor, A model for the boundary condition of a porous material. Part 1, J. Fluid Mech. 49, 319 (1971).

[40] J. L. Auriault, On the domain of validity of Brinkman's equation, Transp. Porous Media 79, 215 (2009).

[41] C. K. W. Tam, The drag on a cloud of spherical particles in low Reynolds number flow, J. Fluid Mech. 38, 537 (1969).

[42] K. Suga, Y. Matsumura, Y. Ashitaka, S. Tominaga, and M. Kaneda, Effects of wall permeability on turbulence, Int. J. Heat Fluid Flow 31, 974 (2010).

[43] G. Neale and W. Nader, Practical significance of Brinkman's extension of Darcy's law, Can. J. Chem. Eng. 52, 475 (1974).

[44] S. Beneddine, D. Sipp, A. Arnault, J. Dandois, and L. Lesshafft, Conditions for validity of mean flow stability analysis, J. Fluid Mech. 798, 485 (2016). 


\section{ABDERRAHAMAN-ELENA AND GARCÍA-MAYORAL}

[45] O. Tammisola and M. P. Juniper, Coherent structures in a swirl injector at $R e=4800$ by nonlinear simulations and linear global modes, J. Fluid Mech. 792, 620 (2016).

[46] S. Dupont, F. Gosselin, C. Py, E. De Langre, P. Hemon, and Y. Brunet, Modelling waving crops using large-eddy simulation: Comparison with experiments and a linear stability analysis, J. Fluid Mech. 652, 5 (2010).

[47] N. Luminari, C. Airiau, and A. Bottaro, Drag-model sensitivity of Kelvin-Helmholtz waves in canopy flows, Phys. Fluids 28, 124103 (2016).

[48] L. Rayleigh, On the stability, or instability, of certain fluid motions, Proc. London Math. Soc. 1, 57 (1879).

[49] C. Scalo, J. Bodart, and S. K. Lele, Compressible turbulent channel flow with impedance boundary conditions, Phys. Fluids 27, 035107 (2015).

[50] N. Tilton and L. Cortelezzi, Linear stability analysis of pressure-driven flows in channels with porous walls, J. Fluid Mech. 604, 411 (2008).

[51] H. B. Squire, On the stability for three-dimensional disturbances of viscous fluid flow between parallel walls, Proc. R. Soc. London Ser. A 142, 621 (1933).

[52] R. D. Cess, A survey of the literature on heat transfer in turbulent tube flow, Westinghouse Research Report No. 8-0529-R24, 1958 (unpublished).

[53] W. C. Reynolds and W. G. Tiederman, Stability of turbulent channel flow, with application to Malkus's theory, J. Fluid Mech. 27, 253 (1967).

[54] J. C. del Álamo and J. Jiménez, Linear energy amplification in turbulent channels, J. Fluid Mech. 559, 205 (2006). 